\begin{tabular}{ccc}
\hline Bentham open & The Open Biomedical Engineering \\
CrossMark & Content list available at: www.benthamopen.com/TOBEJ/ & $\begin{array}{c}\text { The } \\
\text { Biomedical } \\
\text { Enginecring } \\
\text { lournal }\end{array}$ \\
\hline
\end{tabular}

\title{
LETTER
}

\section{Path Loss Measurement and Channel Modeling with Muscular Tissue Characteristics}

\author{
Yu-Ping Qin ${ }^{\mathrm{a}, \dagger}$, Shuang Zhang ${ }^{\mathrm{a}, \mathrm{b}, \mathrm{c}, \mathrm{d}, \dagger}$, Hai-Yan Liu ${ }^{\mathrm{e}}$, Yi-He Liu ${ }^{\mathrm{a},{ }^{*} \dagger}$, You-Zhi Li ${ }^{\mathrm{a}}$, Xue Peng ${ }^{\mathrm{a}}$, Xiu Ma ${ }^{\mathrm{a}}$, \\ Qi-Li Li ${ }^{\mathrm{a}}$ and Xuan Huang ${ }^{\mathrm{a}}$ \\ ${ }^{a}$ The Engineering \& Technical College, Chengdu University of Technology, Leshan, 614000, China \\ ${ }^{b}$ College of Computer Science, Neijiang Normal University, Neijiang, 641000, P.R. China \\ ${ }^{c}$ The Department of Electrical and Computer Engineering, Faculty of Science and Technology, University of Macau, \\ Macau SAR 999078, China \\ ${ }^{d}$ The State Key laboratory of Analog \& Mixed-signal VLSI, University of Macau, Macau SAR 999078, China \\ ${ }^{e}$ Pengan County People's Hospital, Nanchong, 638285, P.R. China
}

Received: August 24, 2016

Revised: November 19, 2016

Accepted: December 15, 2016

\section{Abstract:}

\section{Background:}

The galvanic coupling intra-body communication has low radiation and strong anti-interference ability, so it has many advantages in the wireless communication.

\section{Method:}

In order to analyze the effect of muscle tissue's characteristics upon the communication channel, we selected the muscle of pig buttock as the experimental sample, and used it to study the attenuation property with the galvanic coupling intra-body communication channel along the parallel direction and the transverse direction relative to the muscular fibre line as well as on the surface of destroyed muscular fibre; the study frequency ranges from $1 \mathrm{kHz}$ to $10 \mathrm{MHz}$.In the isotropic experiment, in order to destroy muscle's fibre characteristics, we grinded the muscle four times, at least five minutes for each time. 0dbm sine-wave signal was input to measure the channel attenuation parameter S21 when the transmitter and the receiver were placed at different positions and different distances $\mathrm{d} 1$ and $\mathrm{d} 2(20 \mathrm{~mm}, 40 \mathrm{~mm}, 60 \mathrm{~mm})$, so as to analyze channel loss.

\section{Conclusion:}

Within the same frequency range and at the same communication distance, the maximum error of channel attenuation was $10 \mathrm{~dB}$; within the same frequency, as the communication distance was increased, the channel attenuation rose gradually, with $4 \mathrm{~dB}$ increased every $20 \mathrm{~mm}$. The conclusion provides the basis for building the theoretical model in the future.

Keywords: Galvanic coupling intra-body communication, Parallel, Transverse, Isotropic, Channel loss.

\section{INTRODUCTION}

The Body Area Network (BAN) [1] is a network attached to human body consisting of a set of miniature portable sensors and a body-borne master station (all called BAN coordinator) capable of communication, such as PDA and cell

\footnotetext{
* Address correspondence to this author at the Engineering \& Technical College, Chengdu University of Technology, Leshan, 614000, China; Tel: (+86)8322343466; E-mail: liu_yihe@163.com

$\uparrow$ Authors who made equal contributions
} 
phone. Each sensor may be worn on one's body or implanted in the body. The body-borne master station is the network management unit and the gateway between BAN and external networks (e.g. 3G, WiMAX and Wi-Fi) [2], which achieves safe transmission and exchange of data. Because these sensors communicate by means of the wireless technology, the BAN is also known as wireless body area network (WBAN).

The intra-body communication [1] is an emerging wireless communication with human body as the communication medium. It avoids the effect of complicated wiring upon the system and is applied to the inaccessible area (e.g. brain, bone marrow, thoracic cavity) for many traditional communication methods. So it lays a foundation for the development of the implantable medical technology. The galvanic coupling intra-body communication [2] has low power consumption and low frequency, in which human body is regarded as a resistor and signals are input in human tissues, and signal transmission is realized by detecting difference of change of human electrical signal.

Human tissue is an important factor affecting the galvanic coupling intra-body communication channel [2 - 4], especially muscular tissue, because most of the signals are transmitted in muscular tissue. A large number of experiments showed $[3,4]$ that, different fibre growth characteristics in the parallel and the transverse directions caused large difference between electrical characteristics of the tissue in the two directions.

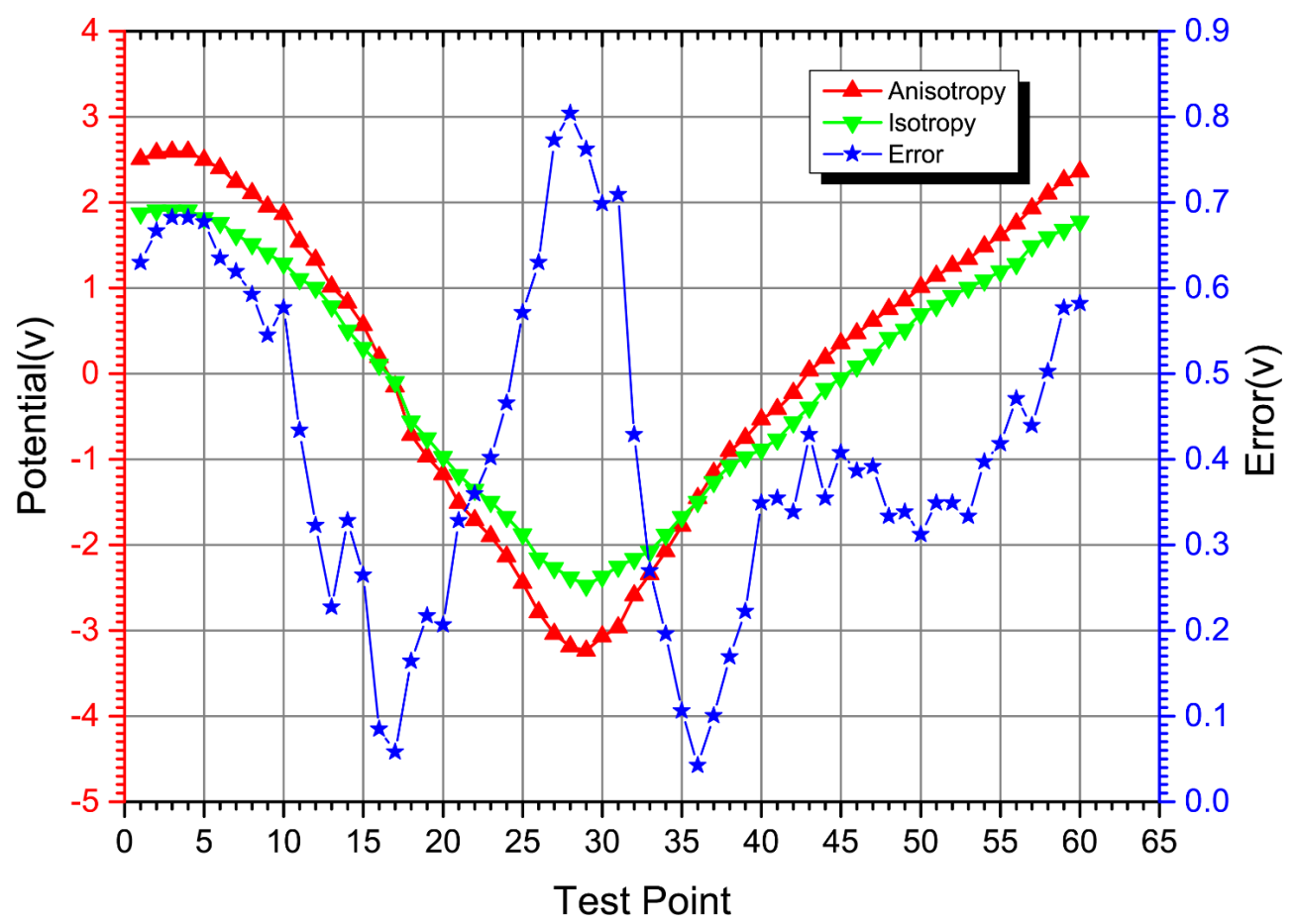

Fig. (1). Effect of human tissue characteristics upon electrical signal [4].

Fig. (1) indicates the voltage values of the test point when the muscle is isotropic or anisotropic. By using the numerical solution method and assuming the tissue in the numerical solution model is human tissue, the electric parameters (conductivity and relative permittivity) of human isotropic or anisotropic muscle layer are brought into the model, and the voltages of test points are detected by providing different test points. After the voltages of the test points are obtained, the effect of muscular characteristics on the detection voltage is analyzed with the absolute error method. It is observed from analysis that, when the detection voltage ranges from $-3 \mathrm{~V}$ to $3 \mathrm{~V}$, the average error caused by the tissue characteristics is up to $30 \%$; therefore, the muscular tissue's characteristics is an important factor in the system modeling, which cannot be neglected.

In order to analyze the effect of muscular tissue characteristics upon channel attenuation, we designed a series of experiments to analyze channel attenuation for muscular isotropy or muscular parallel and transverse anisotropy under the same measurement condition.

\section{MEASURING METHOD PROCEDURE}

Because pigs' tissue characteristics fairly approximate human body's, we selected a piece of fresh pig buttocks 
muscle (died within five hours) as the experimental subject. Prior to the experiment, we removed the fat layer and the skin layer out of the muscle through medical stripping. According to muscle's growth characteristic, a piece of pork without inside crossed fat line was cut and used as the experimental sample. Then the measuring electrodes were set as per some rules (Figs. 2 and 3). Next, the Experiment 1 was completed and the experimental parameters are listed in (Table 1).

To analyze the effect of differences between muscle's isotropy and anisotropy on the communication system, after Experiment 1 was finished, the muscle sample was grinded with the meat grinder to destroy muscle's fibre characteristics. In this experiment, we grinded the muscle four times, not less than five minutes for each time. After grinding was completed, the muscle became pasty. This made the entire piece of muscle to completely lose the muscle fibre characteristics (in normal meat-grinding, meat is required to be grinded for only one time. At that time, muscle was granulated so that its fibre characteristics cannot be destroyed). Finally, the attenuation of the communication channel with isotropic muscular tissue was verified with the grinded meat paste.

The meat paste was filled in a polycarbonate box with the size of $160 \mathrm{~mm} \times 160 \mathrm{~mm} \times 70 \mathrm{~mm}$ (see Fig. 4). In order to prevent bubble from being generated during filling, the box was filled and compacted piece by piece to so that the muscles take geometrical shape; Agcl electrodes were fixed on the surface (see Fig. 1) and Experiment 2 was performed.

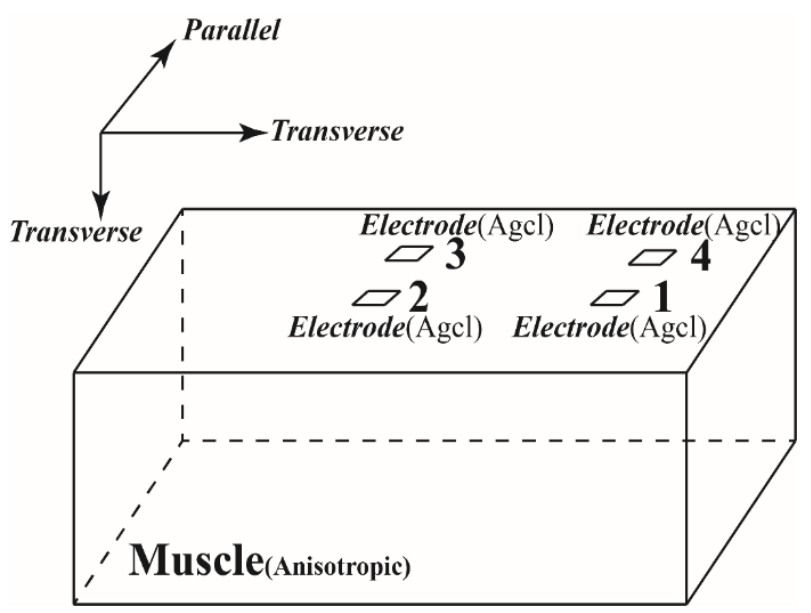

Fig. (2). Muscle cut rule and experiment layout.

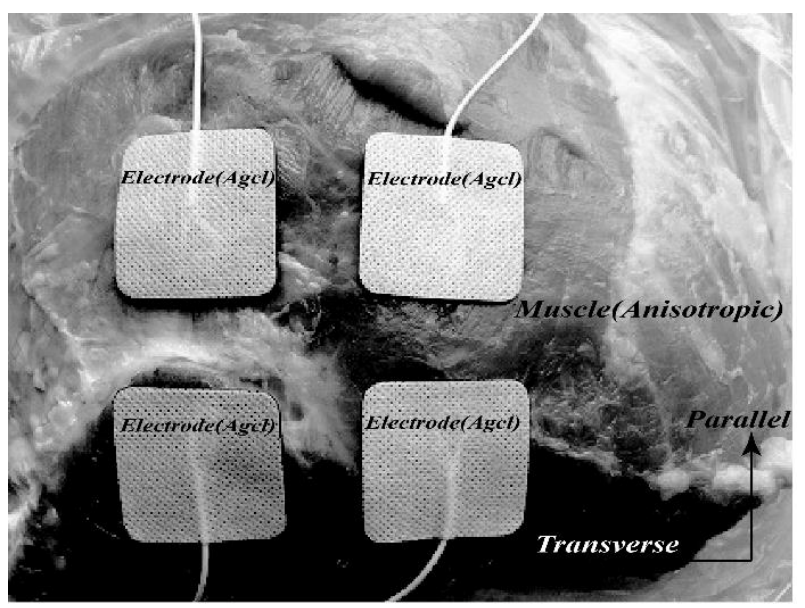

Fig. (3). Experimental layout for anisotropic. 


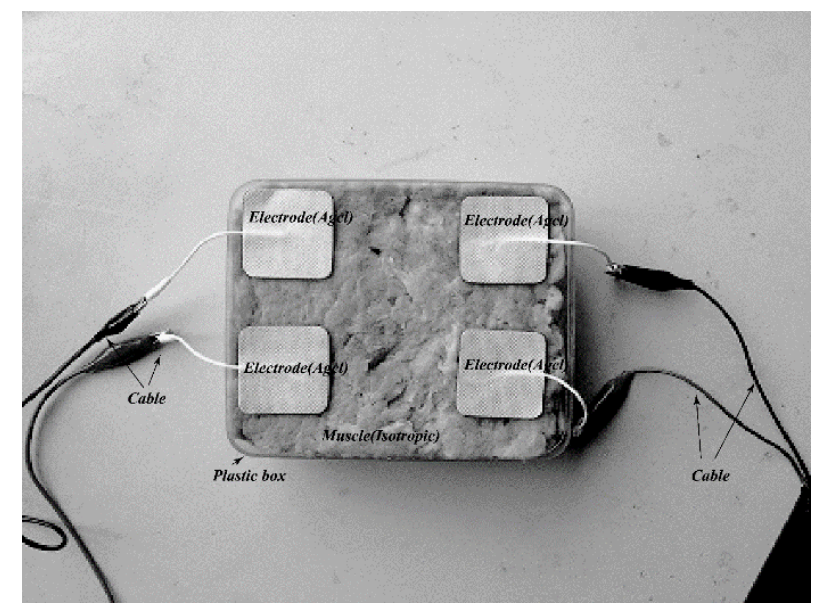

Fig. (4). Experiment for isotropic muscle.

In the experiment, we selected $20 \mathrm{~mm}$ x $20 \mathrm{~mm}$ Agcl physiological electrodes as the experimental electrodes. The minimum distance from electrode edge to sample edge was $10 \mathrm{~mm}$ and the distance between electrodes were $d_{1}, d_{2}$ respectively (see Fig. 5). In the experiment, we used a network analyzer (4395A, Agilent Technologies, Santa Clara, California, USA) and a differential probe (1141A, Agilent Technologies, Santa Clara, California, USA; 1142A, Agilent Technologies, Santa Clara, California, USA). The network analyzer was used to measure channel attenuation and the differential probe was utilized to solve the common-ground problem of devices.

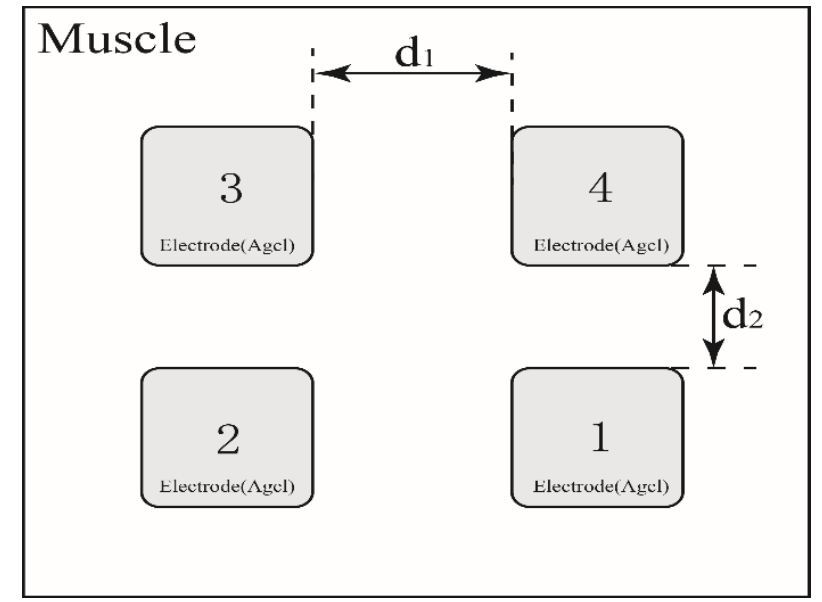

Fig. (5). Layout of experimental electrodes.

\section{MEASUREMENT}

In the experiment, signal attenuation on the muscle surface was measured by changing positions of the transmitting electrode and the receiving electrode; the parameters details are in Table 1 . The $1^{\text {st }}$ to $3^{\text {rd }}$ groups of signals were used to study channel attenuation when measured along the direction parallel to the muscular fibre; the $4^{\text {th }}$ to $6^{\text {th }}$ groups of signals were measured along the direction transverse to the muscular fibre. The $7^{\text {th }}$ to $9^{\text {th }}$ groups of signals were utilized to analyze the attenuation of the isotropic channel when the filler was meat paste. In the measurement process, channel attenuation was measured from (TX) to (RX) within the frequency range of $1 \mathrm{kHz}$ to $10 \mathrm{MHz}$ by means of the $\mathrm{B} / \mathrm{R}$ function (The forward direction transmission property of the tested piece) of the network analyzer; meanwhile, the signal source was $0 \mathrm{dbm}$ sine-wave signal. Moreover, by changing the positions of the communication distance $(20 \mathrm{~mm}$, $40 \mathrm{~mm}$ and $60 \mathrm{~mm}$ ) as well as the transmitting and the receiving electrodes, the channel attenuation parameter S21 was measured and the path loss of the channel was analyzed.

\section{RESULTS AND ANALYSIS}

Fig. (6) shows all results of this experiment. In the experiment, the transmitters $1(+)$ and $2(-)$ and the receivers 4 
$(+)$ and $3(-)$ were arranged. At this moment, the signal was transmitted along the direction from the $1^{\text {st }}$ to $4^{\text {th }}$ and the transmitting direction was parallel to the muscle fibre. It can be found from Fig. (6). that, when the signal carrier frequency was $1 \mathrm{kHz}$ to $300 \mathrm{kHz}$, the channel attenuation gradually increased as the carrier frequency increased when the signal carrier frequency was $300 \mathrm{kHz}$ to $10 \mathrm{MHz}$, the channel attenuation gradually decreased as the carrier frequency reduced. When the transmitters $2(+)$ and $3(-)$ and the receivers $1(+)$ and $4(-)$ were arranged, at this moment, the signal was transmitted along the direction from $2^{\text {nd }}$ to $1^{\text {st }}$, and the signal transmitting direction is transverse to muscle fibre. It can be found from Fig. (6) that, when the signal carrier frequency was $1 \mathrm{kHz}$ to $2 \mathrm{MHz}$, the channel attenuation gradually increased as the carrier frequency increased; when the signal carrier frequency was $2 \mathrm{MHz}$ to $10 \mathrm{MHz}$, the channel attenuation gradually decreased as the carrier frequency reduced.

Table 1. Measured parameters.

\begin{tabular}{|c|c|c|c|c|c|c|}
\hline \multirow[t]{2}{*}{ Times } & \multicolumn{2}{|c|}{ Transmitter } & \multicolumn{2}{|c|}{ Receiver } & \multirow{2}{*}{$\begin{array}{c}\mathrm{d} 1 \\
(\mathrm{~mm})\end{array}$} & \multirow{2}{*}{$\begin{array}{c}\text { d2 } \\
(\mathrm{mm})\end{array}$} \\
\hline & $\begin{array}{c}\text { Electrode } \\
(+)\end{array}$ & Electrode (-) & Electrode (+) & Electrode (-) & & \\
\hline 1 & 1 & 2 & 4 & 3 & 20 & 20 \\
\hline 2 & 1 & 2 & 4 & 3 & 20 & 40 \\
\hline 3 & 1 & 2 & 4 & 3 & 20 & 60 \\
\hline 4 & 2 & 3 & 1 & 4 & 20 & 20 \\
\hline 5 & 2 & 3 & 1 & 4 & 40 & 20 \\
\hline 6 & 2 & 3 & 1 & 4 & 60 & 20 \\
\hline 7 & 2 & 3 & 1 & 4 & 20 & 20 \\
\hline 8 & 2 & 3 & 1 & 4 & 40 & 20 \\
\hline 9 & 2 & 3 & 1 & 4 & 60 & 20 \\
\hline
\end{tabular}

The occurrence of the phenomenon was found to be depended on the electrophysiological characteristics of muscle fibre in the parallel and the transverse directions. When the signal carrier frequency was $1 \mathrm{kHz}$ to $300 \mathrm{kHz}$, muscle's electric conductivity in the parallel and the transverse directions gradually increased as the frequency rose; muscle tissue's conductivity improved and signal diffused increasingly so as to make the signal not to be concentrated around the electrode effectively, while the signal quickly diffused throughout the entire muscle mass. The channel attenuation was supposed to reduced, however, muscle tissue's electric characteristics highly differed in the parallel direction and the transverse direction [3], and signal diffuse speed also differed greatly in the two directions, thus, the measured channel attenuation was increased as the frequency ascended.

When the signal carrier frequency increased from $300 \mathrm{kHz}$ to $10 \mathrm{MHz}$, the tissue's permittivity decreased and the tissue's capacitance characteristics became quite obvious. At the moment, the distinct electrical resistance property of the tissue gradually changed to the capacitance characteristics, and the skin effect became more and more distinct. Because the transmission distance was relatively short, the capacitance characteristics predominated in the entire system as the frequency rose. In order to verify the above thought, we designed the isotropic experiment; in the experiment, we destroyed the fibre characteristics of a piece of pork, and then the meat paste without fibre characteristics was used to re-build the geometrical model; so a cube isotropic pork model was obtained. And then channel attenuation was analyzed by inputting $0 \mathrm{dbm}$ sine-wave signal.

It could be found from Fig. (6). that, when the signal carrier frequency ranged from $1 \mathrm{kHz}$ to $10 \mathrm{MHz}$, because of no effect of muscle fibre characteristics, the electric conductivity was improved as the frequency increased, while channel attenuation became smaller as the frequency increased. Within the frequency range of $300 \mathrm{kHz}$ to $10 \mathrm{MHz}$, because of the effect of the capacitance effect, at the moment, the capacitance effect and electrical resistance property of the channel acted on the overall channel jointly; but the capacitance effect became more and more obvious with the increase of the frequency, so as to cause further sharp reduction of the channel attenuation within the frequency domain. 


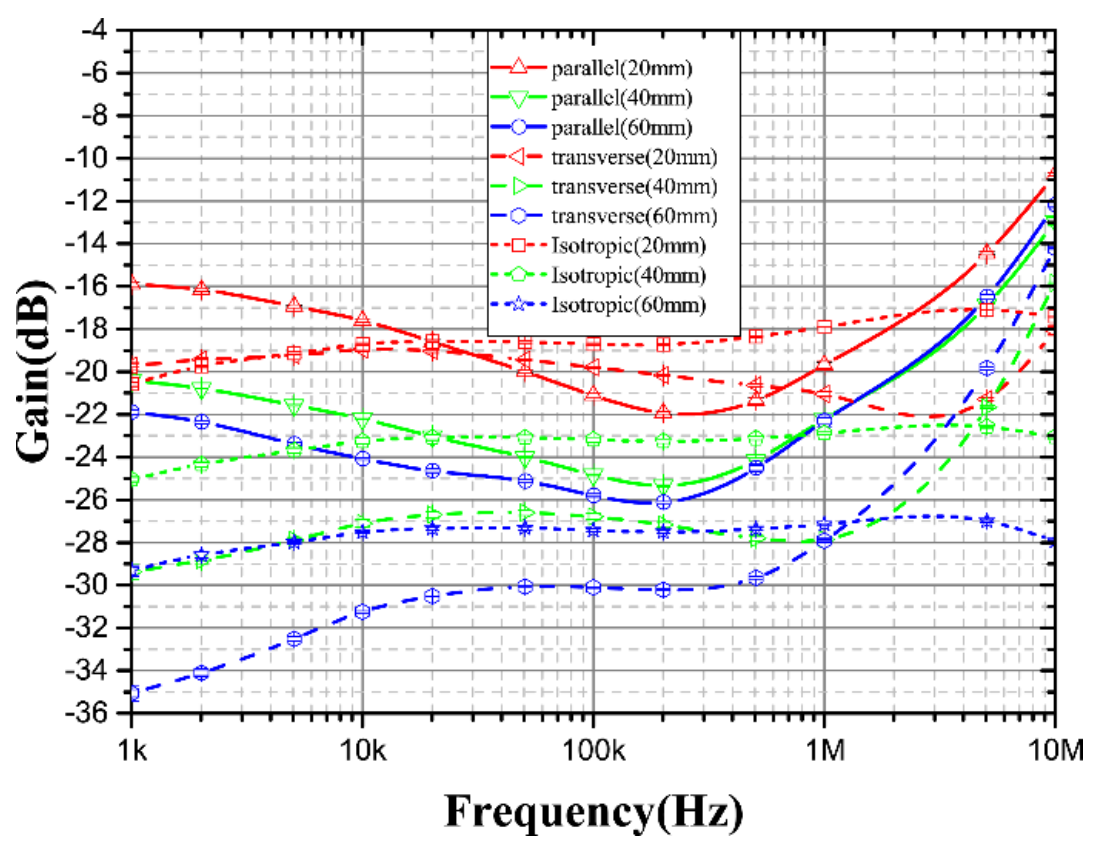

Fig. (6). Experiment results $(\mathrm{d}=20 \mathrm{~mm}, 40 \mathrm{~mm}, 60 \mathrm{~mm})$.

In the measurement, we also changed the communication distance between the transmitter and the receiver. The experiment showed that, whether in the isotropic communication channel or in the anisotropic communication channel, the attenuation was increased as the distance rose, with about $4 \mathrm{~dB}$ added every $20 \mathrm{~mm}$. This was consistent with the results in Zhang et al. [2, 4].

\section{Modeling Complex Channel Attenuation Model}

To interpret the above experimental phenomena, we built the channel attenuation model at the static state by means of the volume conductor theory. On the basis of the Maxwell Equation at the static state, the following can be derived:

$$
\nabla \bullet \sigma(f) \nabla \varphi \approx 0
$$

where $\sigma(f)$ indicates the tissue's conductivity at the frequency of $f$, and $\phi$ is the conductor 's coupling potential.

Assume the muscle's conductivities in the parallel and the traverse directions are $\sigma_{\mathrm{t}}(f)$ and $\sigma_{\mathrm{l}}(f)$ at the frequency of $f$. The isotropic conductivity can be given as follows based on the references:

$$
\sigma(f)=\sqrt{\sigma_{t}(f) \sigma_{l}(f)}
$$

The measured results show that, channel attenuation quantities of muscle tissue in the traverse and the parallel directions may be denoted by $G_{T}$ and $G_{P}$, respectively $G_{C}$; indicates the channel attenuation of meat paste with fully destroyed fibre characteristics. Assuming the damage factor of the muscular tissue $\delta$ is (muscle's damage degree $0<\delta<$ 1), the moisture loss factor is $0<\varepsilon<1$, the following composite channel attenuation model can be derived based on Equation (1) and Equation (2):

$$
G_{C}=-\sqrt{k \cdot G_{T} \cdot G_{P}}
$$

where $k=\delta(1-\varepsilon)$. 


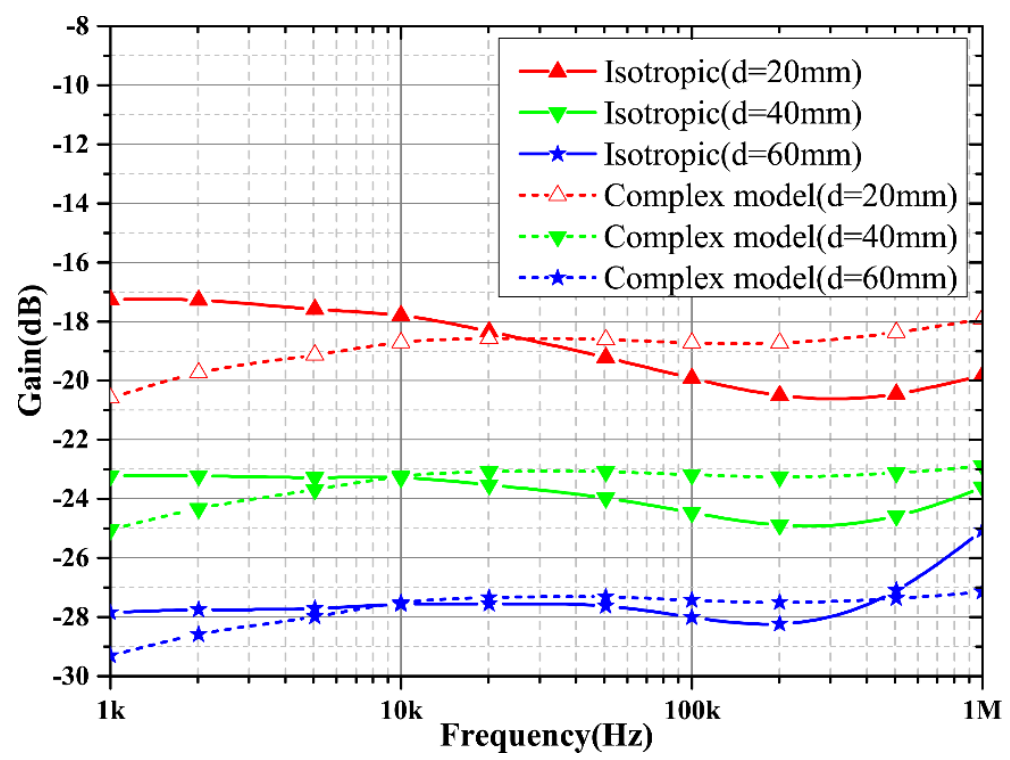

Fig. (7). Complex attenuation model and isotropic experimental results.

Fig. (7) shows the contrast between the composite model and the isotropic experiment. In the model, tissue fibre is assumed to be destroyed, namely; $\delta=1$ the moisture loss is zero in the destroying process, namely $k=1$. By comparing the model result and the experimental result, it can be seen that the variation tendency in the composite model's calculated result is almost identical to that of the isotropic experimental result. However, there are certain differences between both results; the shorter the distance is, the larger the error is. This is because the galvanic coupling intra-body communication is greatly affected by the capacitance characteristics in the short-distance communication $(\mathrm{d}=20 \mathrm{~mm})$; so the error is relatively large. When the communication distance is large $(d=60 \mathrm{~mm})$, the calculated result and the experimental result of the model are almost the same. This is because the influence of the transmitting electrodes' capacitance effect upon the receiving electrodes is small, so both results are nearly in accordance with eacth other

\section{CONCLUSION}

In this experiment, we selected pig buttocks muscles as the sample to measure attenuation characteristics of the galvanic coupling intra-body communication along the parallel direction and the transverse direction relative to the muscular fibre line as well as on the surface of destroyed muscular fibre within the frequency range of $1 \mathrm{kHz}$ to $10 \mathrm{MHz}$. In the isotropic experiment, in order to destroy muscle's fibre characteristics completely, we grinded the muscle four times, at least five minutes for each time. 0dbm sine-wave signal was input to measure the channel attenuation parameter S21 at different positions of transmitting and receiving electrodes and at different distances d1 and d2 $(20 \mathrm{~mm}, 40 \mathrm{~mm}, 60 \mathrm{~mm})$, so as to analyze channel loss property. Within the same frequency range and at the same communication distance, the maximum error of channel attenuation was $10 \mathrm{~dB}$ because of different tissue characteristics; within the same frequency, as the communication distance grew, the channel attenuation was gradually raised, with $4 \mathrm{~dB}$ increased every $20 \mathrm{~mm}$. Therefore, in building the theoretical analytical model, we should consider the effect of the distance on the channel transmission as well as tissue characteristics' effect, so as to provide the basis for building the theoretical model later.

\section{CONFLICT OF INTEREST}

The authors confirm that this article content has no conflict of interest.

\section{ACKNOWLEDGEMENTS}

This work presented in this paper is supported by the Science and Technology Development Fund of Macau (FDCT) under 087/2012/A3, \& 047/2013/A2; the Research Committee of the University of Macau under Grants 063/2009/A, MYRG079(Y1-L2)-ST12-VMI, MYRG103(Y1-L3)-FST13-VMI, MRG014/MPU/2014/FST, and MYRG2014-00010-AMSV; the Key Fund Project of Sichuan Provincial Department of Education under Grant13ZA0003, Grant 14ZB0360, Grant 14ZB0363, Grant 14ZB0352; The Sichuan Province Department of Science 
and Technology under Grant 2015JY0119, the Key Fund Project of Leshan Science and Technology Bureau (15ZDYJ0177). The Engineering and Technical College of Chengdu University of Technology under Grants (C122016030). The Leading talent training project of Neijiang Normal University (2016[Liu Yi-He]).

\section{REFERENCES}

[1] T.G. Zimmerman, "Personal area Networks (PAN): Near-field Intrabody Communication", Master thesis, Massachusetts Institute of Technology, MA, USA, 1995.

[2] S. Zhang, S.H. Pun, P.U. Mak, Y-P. Qin, Y-H. Liu, and M.I. Vai, "Communication channel modeling of human forearm with muscle fiber tissue characteristics", Technology and Health Care, vol. 24, no. 5, pp. 681-687, 2016. [http://dx.doi.org/10.3233/THC-161219] [PMID: 27233089]

[3] F.L.H. Gielen, "Electrical conductivity of skeletal muscle tissue: experimental results from different muscles in vivo", Medical \& Biological Engineering \& Computing., vol. 22, pp. 569-577, 1984.

[4] W He, Q Wu, L Liu, H Yang, and H Liu, "Effects of human thorax tissues on conduction of electrocardiogram and body surface potential", Journal of Biomedical Engineering/Shengwu Yixue Gongchengxue Zazhi., vol. 16, no. 4, pp. 471-476, 2006.

C) Qin et al.; Licensee Bentham Open

This is an open access article licensed under the terms of the Creative Commons Attribution-Non-Commercial 4.0 International Public License (CC BY-NC 4.0) (https://creativecommons.org/licenses/by-nc/4.0/legalcode), which permits unrestricted, non-commercial use, distribution and reproduction in any medium, provided the work is properly cited. 